\title{
Hadronic resonances as probes of the fireball evolution in heavy-ion collisions at the LHC
}

\author{
Enrico Fragiacomo* (for the ALICE Collaboration) \\ INFN Trieste \\ E-mail: enrico.fragiacomodts.infn.it
}

\begin{abstract}
Hadronic resonances provide valuable observables for the properties of the hot and dense hadronic phase of the fireball created in heavy-ion collisions, since their lifetimes, of the order of few $\mathrm{fm} / c$, are comparable to the time span between the chemical and the kinetic freeze-out, which characterize the latest stage of the fireball evolution. Re-scattering of decay products and regeneration via pseudo-elastic hadron scattering can alter their yields from the values that would be measured in elementary (pp) collisions and those that would be expected from statistical particle-production models. The relative strengths of re-scattering and regeneration, as well as the lifetime of the hadronic phase, can be studied through measurements of resonance yields and their ratios to the yields of long-lived hadrons. An overview of recent results on resonance production from the ALICE experiment is presented for $\mathrm{pp}, \mathrm{p}-\mathrm{Pb}$, and $\mathrm{Pb}-\mathrm{Pb}$ collisions and compared with statistical model predictions.
\end{abstract}

The European Physical Society Conference on High Energy Physics 22-29 July 2015

Vienna, Austria

${ }^{*}$ Speaker. 


\section{Introduction}

In ultrarelativistic heavy-ion collisions a hot and dense state of matter, the quark-gluon-plasma (QGP), is expected to be produced within a volume called the fireball. Shortly after its production, the QGP fireball undergoes a hydrodynamic expansion under its own pressure and cools while expanding [1]. At a critical temperature of about $156 \mathrm{MeV}$ a cross-over transition takes place which confines the quarks into the hadrons (chemical freeze-out). Thermal models successfully predict the yields of the hadrons at the chemical freeze-out [2]. After chemical freeze-out, the (still) hot and dense hadronic fireball keeps expanding and cooling until the so-called kinetic freeze-out. In the hadronic fireball hadrons can interact via elastic and pseudo-elastic scattering causing a change in their momentum.

Resonances having lifetimes comparable to that of the fireball (in the range of few $\mathrm{fm} / \mathrm{c}$ to some tens of $\mathrm{fm} / \mathrm{c}$ ) are sensitive probes for the evolution of the fireball between the chemical and the kinetic freeze-out [3]. In fact, since a fraction of the resonances decay in the fireball and their decay particles are subjected to interaction, the final recostructible resonance yields depend not only on the chemical freeze-out conditions but also on the scattering cross section of the resonance decay particles and the time span between the chemical and the kinetic freeze-out, which controls the fraction of undetected particles. Provided the scattering cross sections of the resonance decay particles are known, an estimate of the duration of the hadronic fireball can be derived by comparing the yields of hadronic resonances to those of long-lived particles [4].

Recent results for the $\phi$ and $\mathrm{K}^{* 0}$ resonances from the ALICE collaboration will be presented. Section 2 presents the experimental setup and the analysis. In Section 3.1, from the comparison of $p_{\mathrm{T}}$ spectra to theoretical models it is shown that a model which relies on thermodynamics and neglects re-scattering effects is unable to fit the data. In Section 3.2, ratios of resonance to longlived particle yields are shown as function of the centrality. Using the model from [3], which correlates the ratio of resonance to long-lived particle yields to the chemical freeze-out temperature and to the time span between the chemical and the kinetic freeze-out, a rough estimate of the latter is derived.

\section{Experimental setup and analysis}

A detailed description of the ALICE detector and of its performance can be found in Refs. [5] and [6]. The analysis presented here relies mainly on the Inner Tracking System (ITS), the Time Projection Chamber (TPC) and the Time Of Flight array (TOF). The tracking system used in this analysis combines the information from the ITS and TPC. The momentum resolution of the TPC is in the range $1-7 \%$ for pions with $1<p_{\mathrm{T}}<10 \mathrm{GeV} / c$. The TPC also provides particle identification (PID) via $\mathrm{d} E / \mathrm{d} x$ measurements. The TPC $\mathrm{d} E / \mathrm{d} x$ measurements allow pions to be separated from kaons for momenta up to $p \sim 0.7 \mathrm{GeV} / c$ and protons from kaons and pions for momenta up to $p \sim 1 \mathrm{GeV} / c$. The TOF allows pions and kaons to be unambiguously identified up to $p \sim 2.5 \mathrm{GeV} / c$. The two mesons can be distinguished from (anti)protons up to $p \sim 4.0 \mathrm{GeV} / c$.

The $\mathrm{Pb}-\mathrm{Pb}$ data at $\sqrt{s_{\mathrm{NN}}}=2.76 \mathrm{TeV}$ and the $\mathrm{p}-\mathrm{Pb}$ data at $\sqrt{s_{\mathrm{NN}}}=5.02$ have been recorded in 2011 and 2013, respectively, using a trigger based on the hit multiplicity recorded by the V0 
detector, which consists of segmented scintillators covering the full azimuth over $2.8<\eta<5.1$ (V0A) and $-3.7<\eta<-1.7$ (V0C). A signal in either V0A or V0C was requested.

Resonances are identified by their main hadronic decay channel $\left(\mathrm{K}^{* 0} \longrightarrow \pi^{ \pm} \mathrm{K}^{\mp}\right.$ and $\phi \longrightarrow \mathrm{K}^{+} \mathrm{K}^{-}$). Due to their very short lifetimes, decay products cannot be distinguished from particles coming from the primary vertex. Their yield is obtained by computing the invariant mass spectrum of all primary candidates and then subtracting a combinatorial background. This is performed by either the event-mixing or the like-sign technique [10]. The signal, after subtracting the combinatorial background, is fitted with a Breit-Wigner plus a polynomial for the residual background. A Voigtian function (convolution of Breit-Wigner function and Gaussian) is used for the extraction of the $\phi$ raw yield. An example of invariant mass spectrum, before and after the subtraction of the event-mixing background, is presented in Figure 1.
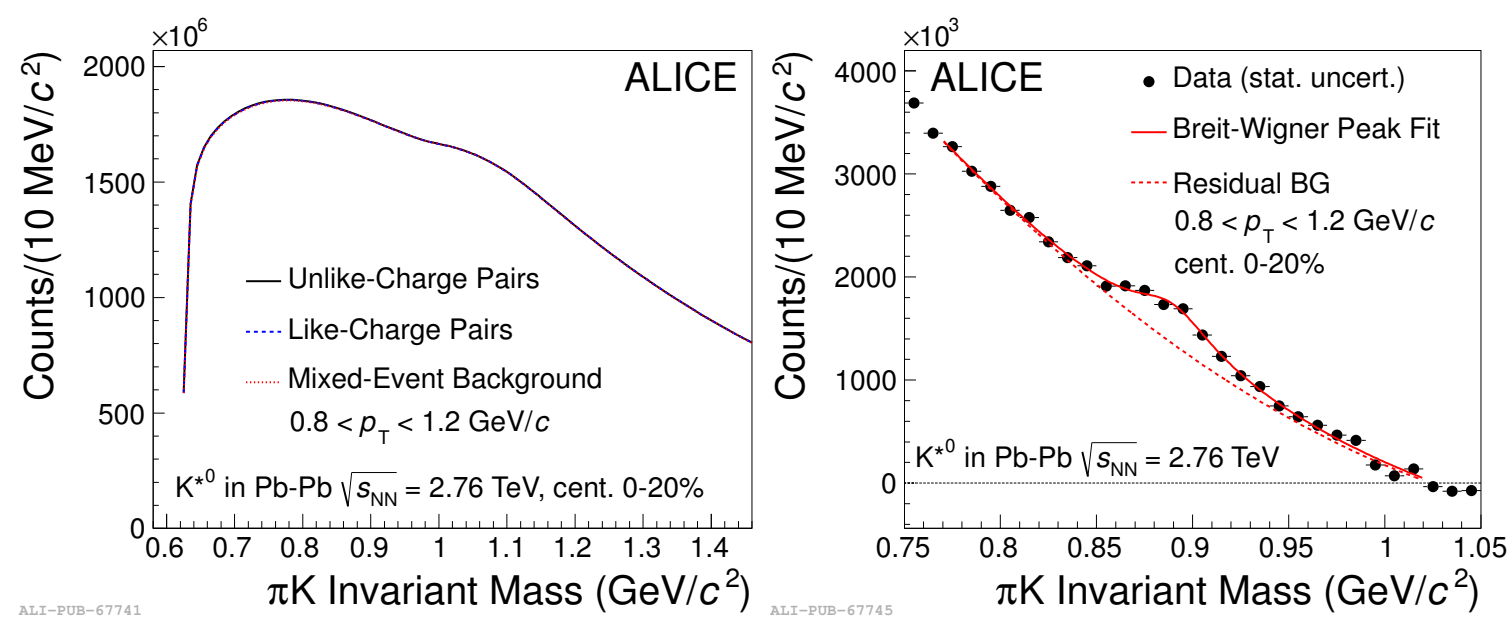

Figure 1: (Left) Example invariant-mass distribution for the $\mathrm{K}^{* 0}$ in the $0-20 \%$ centrality interval and in the $p_{\mathrm{T}}$ range $0.8<p_{\mathrm{T}}<1.2 \mathrm{GeV} / c$. (Right) Invariant-mass distribution after subtraction of the mixed-event background with the fit to describe the peak (solid curve) and the residual background (dashed curve) of the $\mathrm{K}^{* 0}$. Only statistical uncertainties are shown.

In order to extract the total production yield, the raw counts are corrected for the decay branching ratio and for the losses due to geometrical acceptance and detector efficiency. These are determined by Monte Carlo simulation, with particle production simulated using PYTHIA [7] or HIJING [8], for $\mathrm{pp}$ and $\mathrm{Pb}-\mathrm{Pb}$ data respectively, and interactions with the ALICE detector simulated using GEANT3 [9]. Final yields are obtained normalizing to the number of inelastic collisions ( $\mathrm{pp}$ collisions) or to the number of analyzed events in a given centrality range ( $\mathrm{p}-\mathrm{Pb}$ and $\mathrm{Pb}-\mathrm{Pb}$ collisions). The trigger efficiency is also taken into account. The analyses of $\mathrm{K}^{* 0}$ and $\phi$ mesons in pp collisions at $\sqrt{s}=7 \mathrm{TeV}$ and in $\mathrm{Pb}-\mathrm{Pb}$ at $\sqrt{s_{\mathrm{NN}}}=2.76 \mathrm{TeV}$ are described in detail in [10] and [11], respectively. 


\section{Results}

\subsection{Transverse momentum spectra}

Figure 2 shows the transverse momentum distribution of $\mathrm{K}^{* 0}$ and $\phi$ resonances in central $(0-20 \%)$ and peripheral $(60-80 \%) \mathrm{Pb}-\mathrm{Pb}$ collisions at $\sqrt{s_{\mathrm{NN}}}=2.76 \mathrm{TeV}$.

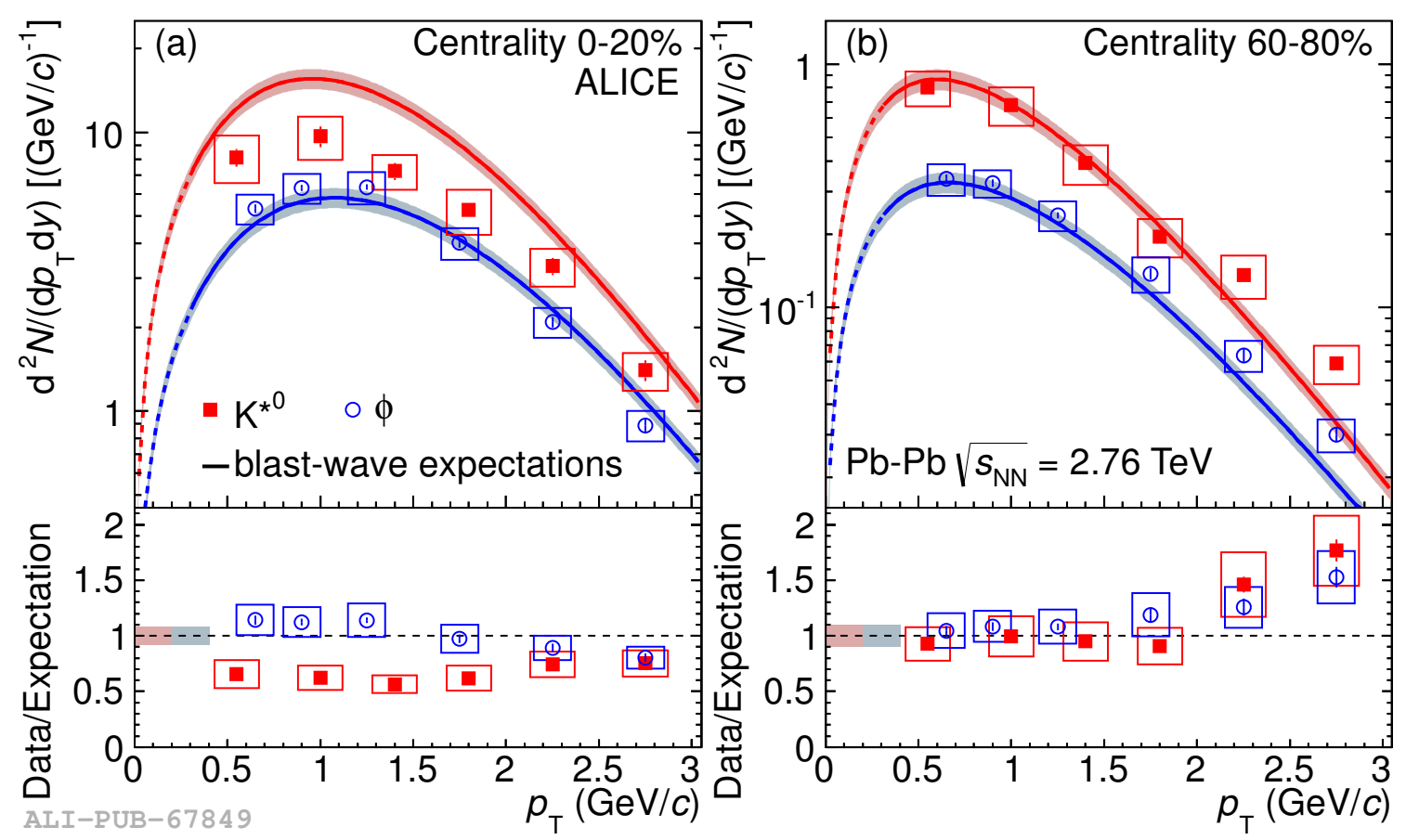

Figure 2: Transverse-momentum distribution of $\mathrm{K}^{* 0}$ and $\phi$ resonances in central (a) and peripheral (b) $\mathrm{Pb}-\mathrm{Pb}$ collisions at $\sqrt{s_{\mathrm{NN}}}=2.76 \mathrm{TeV}$, compared to the blast-wave expectation (see text). The lower panels show the ratios of the measured distributions to the prediction.

The curves in Figure 2 are predictions from a blast-wave model [12] whose parameters, namely the freeze-out temperature $T_{\text {kin }}$, the average transverse velocity $\left\langle\beta_{\mathrm{T}}\right\rangle$ and the exponent of the velocity profile $n$, are obtained from a simultaneous fit to the $p_{\mathrm{T}}$-distributions of charged particles (pions, kaons and protons) in $\mathrm{Pb}-\mathrm{Pb}$ collisions at the same collision energy [13]. The curve for the $\mathrm{K}^{* 0}(\phi)$ is normalized so that its integral equals the product of the measured yield of $\mathrm{K}^{-}$in $\mathrm{Pb}-\mathrm{Pb}$ collisions [13] and the $\mathrm{K}^{* 0} / \mathrm{K}(\phi / \mathrm{K})$ ratio given by a thermal-model fit to ALICE data [2], with a freeze-out temperature of $156 \mathrm{MeV}$.

In the $p_{\mathrm{T}}$ range less than $2 \mathrm{GeV} / c$ the blast-wave prediction gives a satisfactory description of the $\phi$ data, in both central and peripheral collisions, and of the $\mathrm{K}^{* 0}$ in peripheral collisions. On the contrary, in central collisions, the $\mathrm{K}^{* 0}$ data appear suppressed by a factor 1.7 with respect to the theoretical predictions. According to UrQMD calculations [14] this suppression can be interpreted as a hint of re-scattering effects. 


\subsection{Particle ratios}

Another signature of re-scattering effects can be observed in the ratio of resonance to longlived particle yield. Figure 3 shows the resonance to kaon yield ratios [11,13] as function of the cubic root of the charged particle multiplicity density $\left(\mathrm{d} N_{\mathrm{ch}} / \mathrm{d} \eta\right)^{1 / 3}$, which to a good approximation is proportional to the system radius [15]. Data are presented for $\mathrm{pp}, \mathrm{p}-\mathrm{Pb}$ and $\mathrm{Pb}-\mathrm{Pb}$ collisions at $\sqrt{s}=7 \mathrm{TeV}$ and $\sqrt{s_{\mathrm{NN}}}=5.02$ and $2.76 \mathrm{TeV}[10,11]$, respectively, and are compared to the prediction of a grand-canonical thermal model [2], which has a chemical freeze-out temperature of $156 \mathrm{MeV}$ and a baryochemical potential of $0 \mathrm{MeV}$ and does not include re-scattering effects.

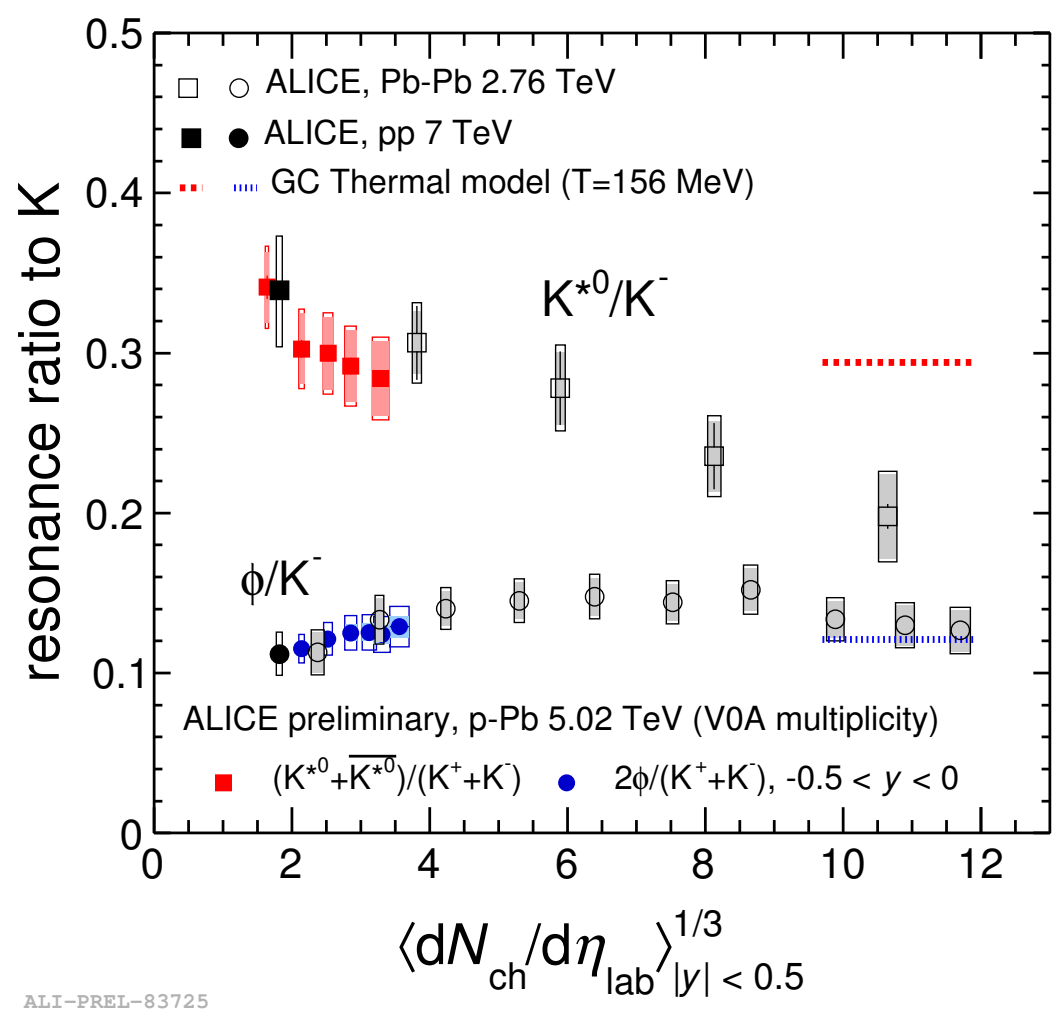

Figure 3: Resonance to kaon yield ratios $[11,13]$ as function of the cube root of the charged particle multiplicity density $\left(\mathrm{d} N_{\mathrm{ch}} / \mathrm{d} \eta\right)^{1 / 3}$ -

The $\phi / \mathrm{K}$ ratio is almost flat for all multiplicity classes from pp to central $\mathrm{Pb}-\mathrm{Pb}$ collisions and it is consistent with the predictions from the thermal model. On the contrary, the $\mathrm{K}^{* 0} / \mathrm{K}^{-}$ratio is consistent with a flat behaviour and in agreement with the theory only for $\mathrm{pp}, \mathrm{p}-\mathrm{Pb}$ and peripheral $\mathrm{Pb}-\mathrm{Pb}$ collisions and exhibits a clear suppression going from peripheral to most central $\mathrm{Pb}-\mathrm{Pb}$ collisions, where the measured ratio is about $60 \%$ of the predicted thermal model value.

Considering the factor of about 10 between the lifetimes of the two resonances, the origin of the differences in the $\mathrm{K}^{* 0}$ and $\phi$ production could be related to a large modification of the $\mathrm{K}^{* 0}$ yield due to the pion rescattering mechanism, which destroys the pion-kaon correlation of the $\mathrm{K}^{* 0}$ decay products. Using the model from [3], which correlates the ratio of resonance to long-lived particle yields to the chemical freeze-out temperature, a lower limit of $2 \mathrm{fm} / c$ for the time span between the chemical and the kinetic freeze-out can be derived. 


\section{References}

[1] L. McLerran, The physics of the quark-gluon plasma, Rev. Mod. Phys. 58 (1986) 1021.

[2] J. Stachel, A. Andronic, P. Braun-Munzinger, and K. Redlich, Confronting LHC data with the statistical hadronization model, Proceedings of Strange Quark Matter 2013 Conference (2013) [arXiv:1311.4662].

[3] C. Markert, G. Torrieri, and J. Rafelski, Strange Hadron Resonances: Freeze-Out Probes in Heavy-Ion Collisions, Proceedings of Pan American Advanced Studies Institute on New States of Matter in Hadronic Interactions (PASI 2002) (2002) [hep-ph/0206260].

[4] S. Singha, B. Mohanty, and Z.-W. Lin, Studying re-scattering effect in heavy-ion collision through $K^{*}$ production, Int. J. Mod. Phys. E 24 (2015) 1550041, [arXiv:1505.0234].

[5] ALICE collaboration, The ALICE experiment at the CERN LHC, J. Instrum. 3 (2008) S08002. DoI link.

[6] ALICE collaboration, Performance of the ALICE Experiment at the CERN LHC, Int. J. Mod. Phys. A 29 (2014) 1430044, [arXiv:1402.4476]. Dor link.

[7] T. Sjóstrand, S. Mrenna, and P. Skands, PYTHIA 6.4 Physics and Manual, J. High Energy Phys. 05 (2006) 026, [hep-ph/0603175]. DOI link.

[8] N. Wang and M. Gyulassy, hijing: A Monte Carlo model for multiple jet production in pp, pA, and AA collisions, Phys. Rev. D 44 (1991) 3501.

[9] R. Brun, F. Carminati, and S. Giani, GEANT detector description and simulation tool, CERN-W5013.

[10] ALICE collaboration, Production of $K^{*}(892)^{0}$ and $\phi(1020)$ in pp collisions at $\sqrt{s}=7 \mathrm{TeV}$, Eur. Phys. J. C 72 (2012) 2183, [arXiv:1208.5717]. Dor link.

[11] ALICE collaboration, $K^{*}(892)^{0}$ and $\phi(1020)$ production in $\mathrm{Pb}-\mathrm{Pb}$ collisions at $\sqrt{s_{N N}}=2-76 \mathrm{TeV}$, Phys. Rev. C 91 (2015) 024609, [arXiv:1404.0495]. Dor link.

[12] S. J. Schnedermann E. and U. Heinz, Thermal phenomenology of hadrons from $200 \mathrm{~A} \mathrm{GeV}$ S+S collisions, Phys. Rev. C 48 (1993) 2462, [nucl-th/9307020]. Dor link.

[13] ALICE collaboration, B. Abelev, Centrality dependence of $\pi, K, p$ production in $P b-P b$ collisions at $\sqrt{s_{\mathrm{NN}}}=2.76$ TeV, Phys. Rev. C 88 (2013) 044910, [arXiv:1303.0737]. DOI link.

[14] M. Bleicher, Relativistic hadron-hadron collisions in the ultra-relativistic quantum molecular dynamics model, J. Phys. G. 25 (1999) 1859.

[15] ALICE collaboration, K. Aamodt, Two-pion Bose-Einstein correlations in central $\mathrm{Pb}-\mathrm{Pb}$ collisions at $\sqrt{s_{N N}}=2.76$ TeV, Phys. Lett. B. 696 (2011) 328-337, [arXiv:1012.4035]. Dor link. 